\title{
Minimal Invasive Technique for Correction of Bowlegs in Children
}

\author{
Ali Zein A. A. Alkhooly \\ Orthopaedic \& Trauma Department, Minima University, El Minia, Egypt. \\ Email: profalizein@yahoo.com \\ Received July $6^{\text {th }}, 2012$; revised July $24^{\text {th }}, 2012$; accepted August $8^{\text {th }}, 2012$
}

\begin{abstract}
Clinical, experimental and histopathological studies were done on sixteen patients and four rabbits. Clinical material includes sixteen patients with bowlegs deformity, their ages ranged between 2 - 7 years and experimental material includes four rabbits each of them 5 weeks of age. Hemicircumferential periosteal release was done on rabbits (only one leg and the other leg is a control one) after ten weeks histopathological study to the proximal tibia was done on the operated and the non operated sides also hemicircumferential periosteal release was done on the patients at the proximal tibia on the medial side. Growth changes occur in the tibia of the experimental animals and histopthological changes were observed at the growth plate adjacent to the divided periosteum and the clinical results on the patients were encouraging and correction of bowlegs occur within $6-8$ months after operation.
\end{abstract}

Keywords: Hemicircumferential; Bowlegs; Periosteum

\section{Introduction}

The close connection and equilibrium between the function of the growth plate and the periosteum in the immature skeleton is well known [1].

The periosteal sleeve stretches from one growth plate to the other, moss in 1972 [2] suggested that tension develops in the periosteum as a consequence of chondrogenesis in the cell columns of the growth plate and this tension may inhibit cell proliferation in cartilage column.

After circumferential division, the periosteum retracts away from the division, so the growth stimulus and increase activity would be maximal at the growth plate adjacent to the divided periosteum [3].

Honghton and Rooker (1979) [4] created valgus angulation in the tibia of rabbit by medial hemicircumferential periosteal division of the upper part of the bone.

In our study we record our early results from such a simple procedure (hemicircumferential periosteal release) in correction of angular deformity of long bone supported by experimental and histopathological study.

\section{Materials}

Our clinical material includes sixteen patients, 11 males and 5 females their ages ranged from 2 - 7 years complaining from bowlegs, two of them with tibia vara and the remaining were deformity after healed rickets.

Experimental material includes four rabbits each of them 5 weeks of age.

\section{Methods}

On experimental study; technique was done on experimental animal (rabbit) on the medial aspect of the upper end of the tibia just medial to the tibial tubercle and hemicircumferential incision of the periosteum was done just below the pesanserinus, then the ends of the cut were separated, then closure of the wound.

Follow up X-ray was done (P/A) on both legs to show the changes on the upper end of tibia, and valgus changes were recorded regularly every 2 weeks till 10 weeks (Figure 1) before operation (Figure 2) after operation.

After 10 weeks the animals were killed and both legs (operated and non-operated) were dissected and cut sections were taken from the upper tibiae of the operated and non-operated one and examined histopathologically.

\section{Clinical Methods}

Under general anaethesia and tourniquet a curved incision was made in the medial aspect of the upper part of tibia just medial to the middle of the tibial tubercle and extended distally about $2 \mathrm{~cm}$ with its convexity towards the medial side of the leg.

Hemicircumferential periosteal division was done just below pesanserinus and immediate diastasis of the cut ends was done. Closure of the wound and the leg was not 


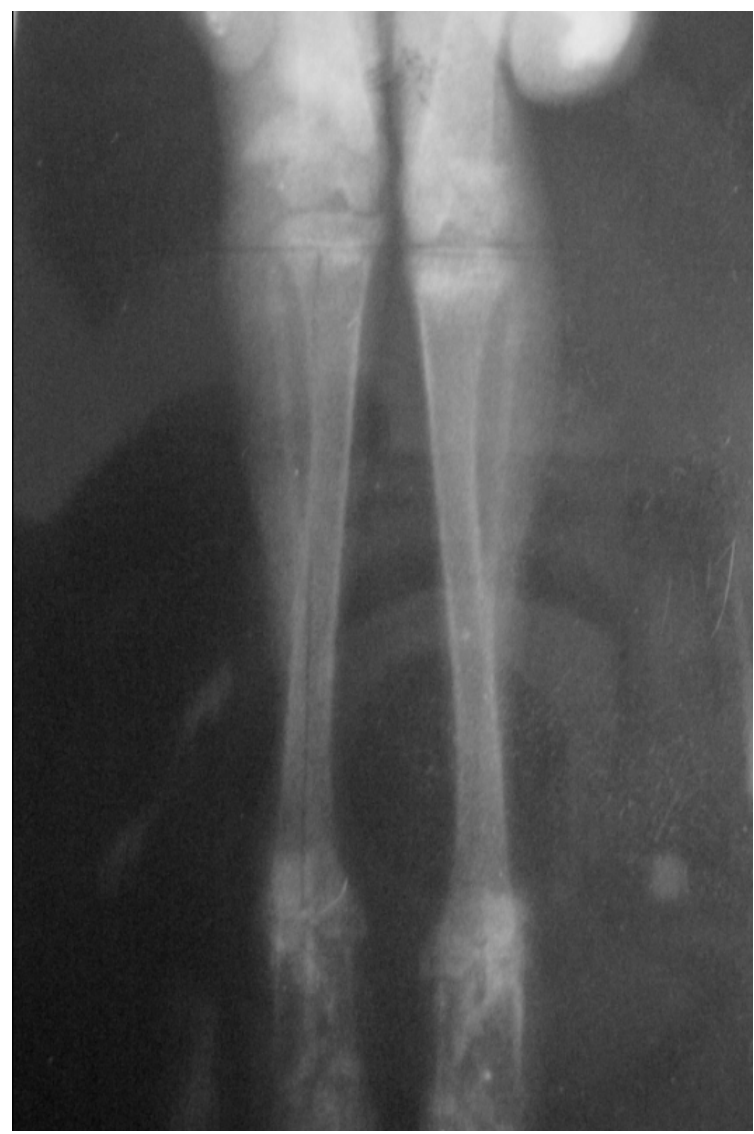

Figure 1. X-ray of both legs of a rabbit before experimental study.

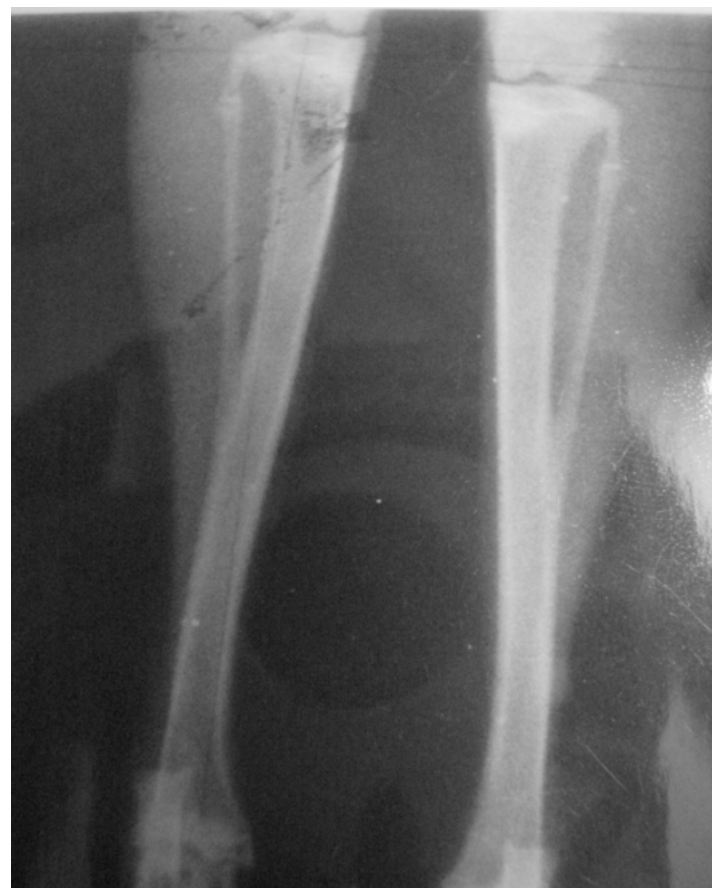

Figure 2. The valgus change in the tibia of operated side (Sshape appearance) and longitudinal over growth in comparison to the non-operated side. immobilized.

Patients walk from the second day of the operation and stitches were removed after one week. Clinical examination of the patient every two months and the degree of improvement were observed.

$\mathrm{X}$-rays to both legs from the knee joint to the ankle joint (P/A) every two months and changes of valgus angle were observed. (Valgus angle is a modification of the angle of deformity assessed by Erkki O. Karaharju et al. in 1976 [5] which equals the difference between axial angle and epiphysis angle increase in the angle means correction of the bow legs)

\section{Results (Experimental)}

There was valgus deformity of the operated tibiae also an S-shape appearance of the tibia of the operated side and longitudinal overgrowth (Figures 2 and 3).

\section{Results (Histopathological)}

By microscopically examination, the endochondral cartilage towards the medial side of the tibia (operated side) was proceeding largely more than the lateral side (nonoperated) of the tibia, there was an increase of the width of the resting zone and there was also an increase in the amount of chondrocyte of the proliferative zone. The chondrocyte lacunae in the maturation zone were croded and elongated more than those of the lateral side (nonoperated side). So there was a marked histological change between the operated and the nonoperated side (Figures 4-6).

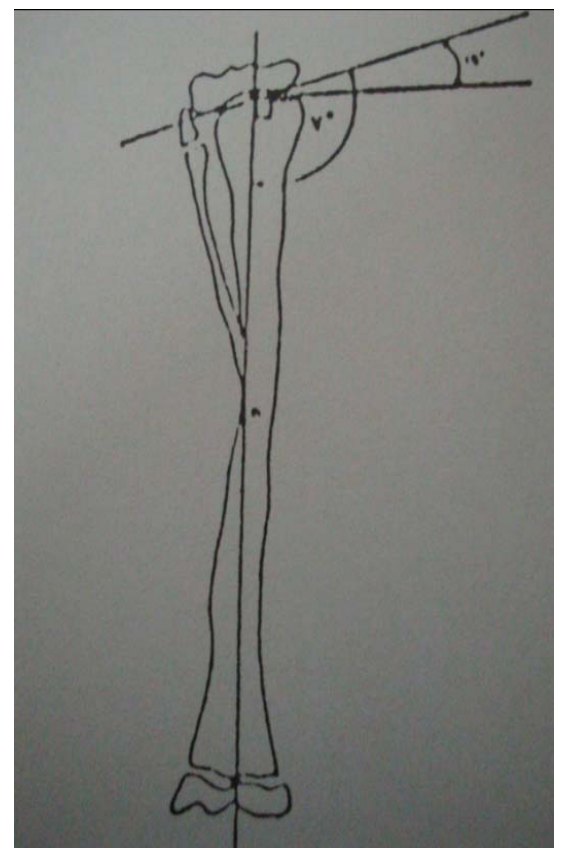

Figure 3. Diagram showing the valgus angle(s) which means difference between angle $v$ and 90 degrees angle. 


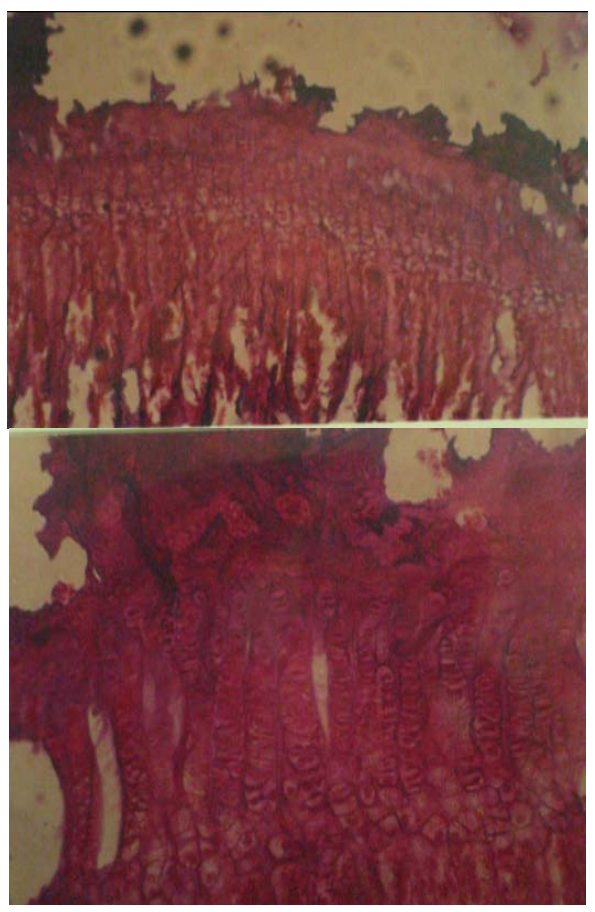

Figure 4. Photomicrograph showing the growth pattern at the lateral part of upper tibial epiphesis in the non-operated tibia before and after ten weeks (control side).

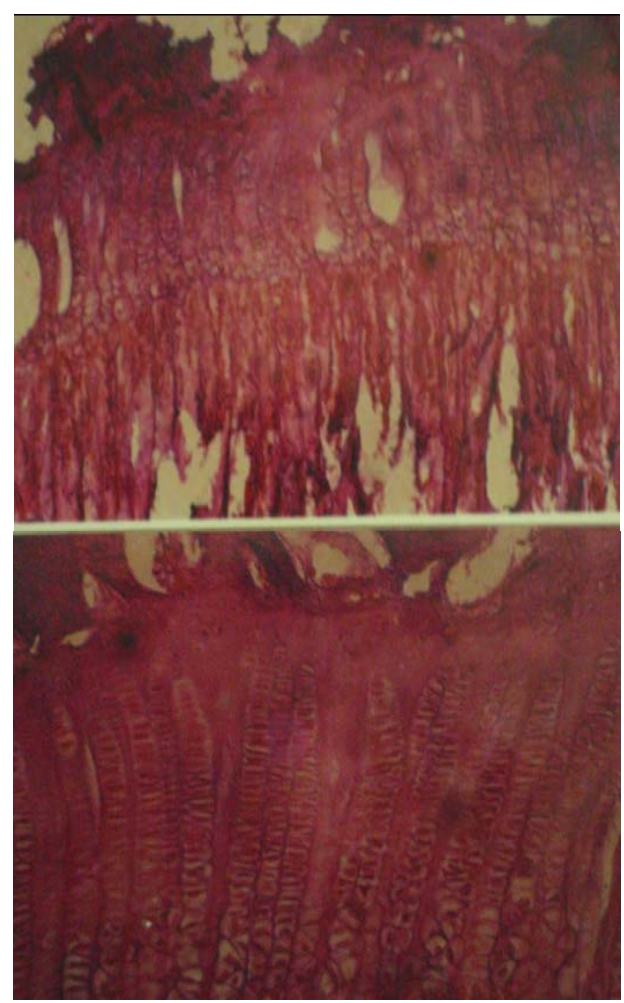

Figure 5. Photomicrograph showing the growth pattern at the medial part of upper tibial epiphesis in the operated tibia before and after ten weeks of study (there is marked increase in the resting zone-proliferative zone and maturation zone) (operated side).

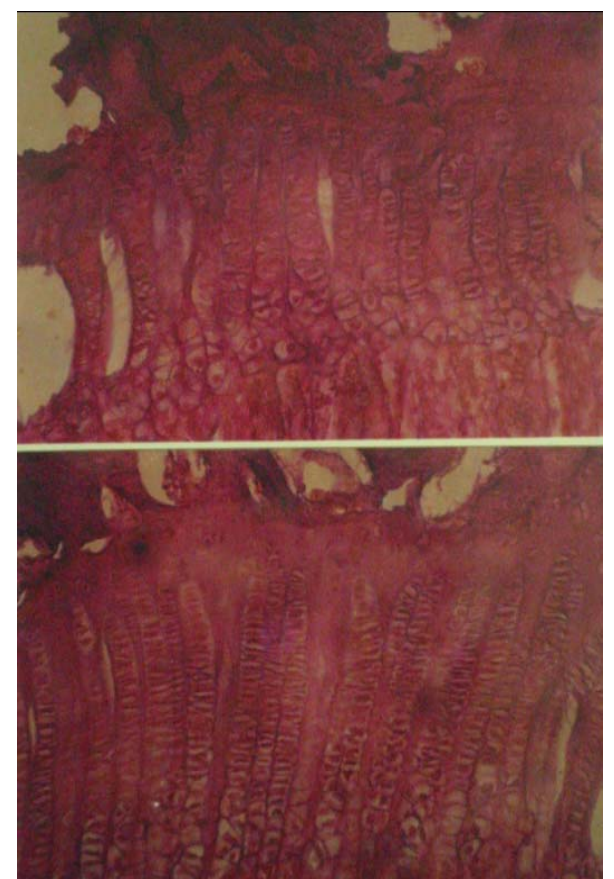

Figure 6. Difference between growth pattern in the control side (above) and the operated side (below).

\section{Results (Patients)}

1) Clinically: maximaum correction occurs within $6-8$ months after operation (Figures $\mathbf{7}$ and $\mathbf{8}$ ).

2) Radiologically: valgus angle was recorded preoperatively and every two months in $(\mathrm{P} / \mathrm{A}) \mathrm{X}$-ray (Figure 9). In cases of tibia vara there were marked improvement in bone density and the fragmentation of upper tibial metaphysis was healed.

3) Functionally: there was a good function of the limb; no pain, limp or stiffness of joint.

\section{Discussion}

The periosteum in young bone is thick and has an inherent elastisity [6] and loosely attached to the diaphysis of the tibia and has a strong connection to the perichondral ring, so exerts a constant mechanical restraint at both ends of the bone [4]. In the growing child the major contribution to longitudinal tibial growth is from the proximal epiphyseal plate, so the periosteal release in the proximal tibial metaphysis will produce the maximum stimulus to the growth [7].

Also it is well known that complete circumferential division of the periosteum stimulate longitudinal growth of bone [8], whether stimulation of the growth plate after division of the periosteum is due to vascular theory, or to mechanical release of the periosteal restrain of the growth plates [9], The mechanical theory, the results of experimental work of Houghton and Rooker [4] reinforce the mechanical theory. 

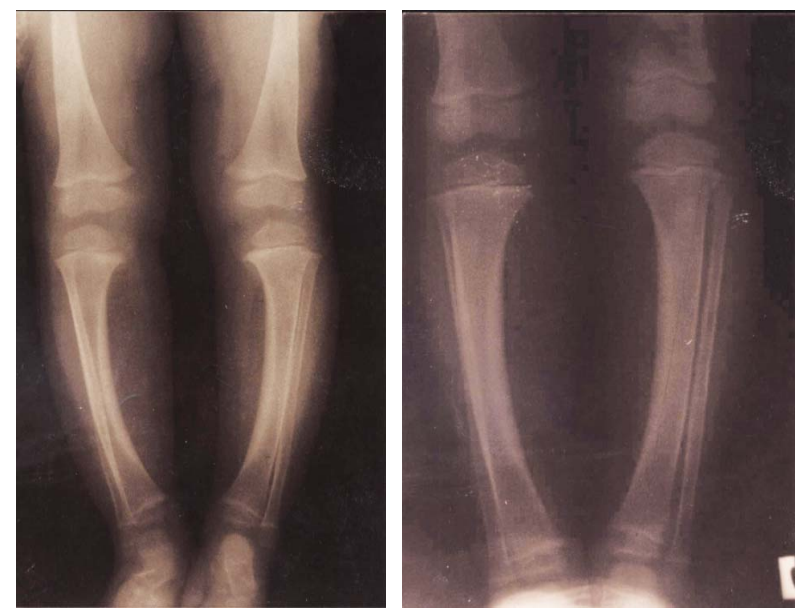

Figure 7. Healed rickets in child aged 6 years before and 8 months after procedure.
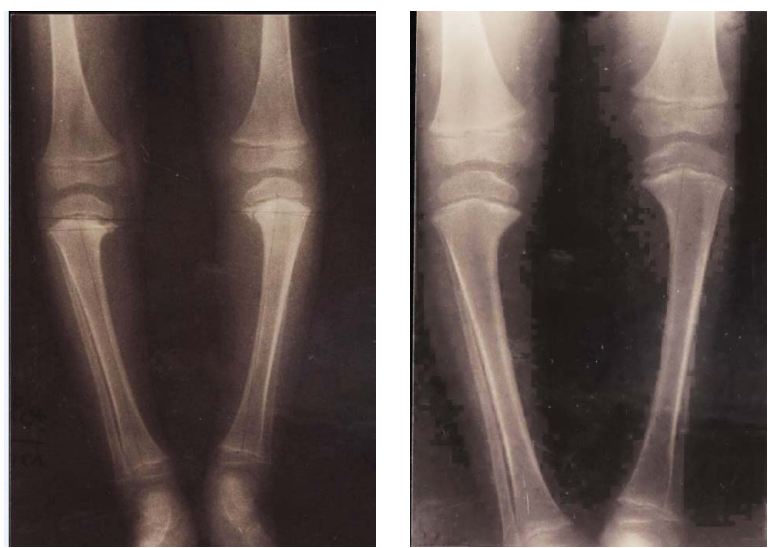

Figure 8. Tibia vara in child aged 4 years before and after 8 months.

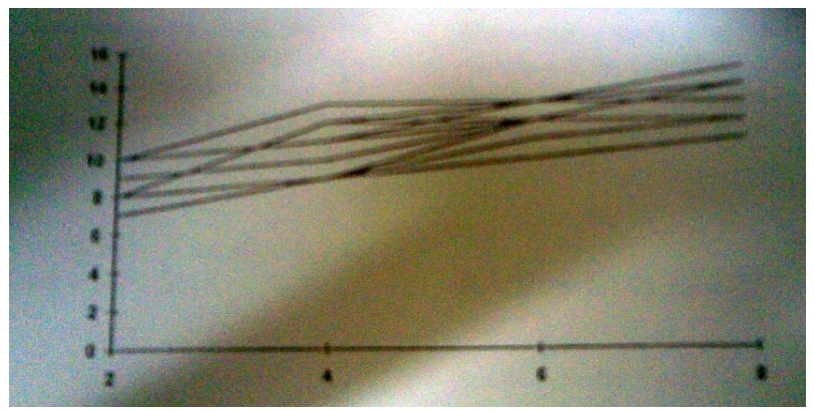

Figure 9. Changes in the valgus angle in degrees every 2 months in operated cases (clinical material).

Periosteal stripping has been used clinically to treat leg length inequality $[10,11]$. In our clinical results there was marked correction of the deformity of legs within $6-8$ months after operation, also the radiological results show increase in the valgus angle. In cases of tibia vara hemicircumferential periosteal release leads to increase vascularity of proximal tibial metaphysis as the fragmentation healed and increase in valgus angle.
There was growth disturbance of the tibia of rabbits after a medial hemicircumferential proximal periosteal division as shown in X-ray and in histopathological examination.

This growth disturbamce of the tibia in the form of valgus deformity, S-shape tibia and longitudinal over growth explained by Pauwel's law which stated that a weight bearing growth plate aligns itself perpendicular to the load [12].

Also hemi circumferential periosteal division leads to microscopically changes in the growth plate of the operated (medial) side than non-operated side.

\section{Conclusions}

1) The periosteum is a fibroelastic membrane stretched between the growth plates and controlling the rate of growth by its inherent tension, and circumferential division of the periosteum, releases this tension and increases rate of growth.

2) Partial periosteal division leads to assymetrical growth acceleration which leads to angular deformity of long bone, so hemicircumferential periosteal division has a place in the treatment of angular deformities of long bone in children.

3) The procedure is simple, easy and safer than major operations for correction of angular deformities such as epiphyseal stapling, epiphysiodesis or corrective osteotomy with their unperidictable results.

\section{Acknowledgements}

Thanks to professor dr. saadia ragab (Histology Department, Faculty of Medicine, El Minia University) also thanks to professor Dr. Salwa Gaber (Pathology Department, Faculty of Medicine, El Minia University).

\section{REFERENCES}

[1] P. Lacroix, "The Organization of Bones," Churchill, London, 1951.

[2] M. L. Moss, "The Regulation of Skeletal Growth," In: R. J. Goss, Ed., The Regulation of Organ and Tissue Growth, Academic Press, New York, 1972, pp. 127-142.

[3] E. Warrel and J. F. Taylor, "The Role of Periosteal Tension in the Growth of Long Bones," Journal of Anatomy, Vol. 128, 1979, pp. 179-184.

[4] G. R. Houghton and G. D. Rooker, "The Role of Periosteum in the Growth of Long Bones. An Experimental Study in the Rabbit," The Journal of Bone \& Joint Surgery, Vol. 61-B, 1979, p. 218.

[5] E. O. Karaharju, A. Soini, et al., "Remodeling by Asymmetrical Epiphyseal Growth: An Experimental Study in Dogs," The Journal of Bone and Joint Surgery, Vol. 58-B, 1976, pp. 122-126.

[6] H. Van Der Sandt, "The Influence of Transverse Section 
in the Periosteum on the Growth of the Rabbit Femur," Thesis, Catholic University, Nijmegen, 1977.

[7] M. C. Lynch and J. F. Taylor, "Periosteal Division and Longitudinal Growth in the Tibia of the Rat," The Journal of Bone and Joint Surgery, Vol. 69-B. No. 5, 1987.

[8] R. G. Crilly, "Longitudinal Overgrowth of the Chicken Radius," Journal of Anatomy, Vol. 112, 1972, pp. 11-18.

[9] G. D. Rooker and R. B. Salter, "Prevention of Valgus Deformity Following Fracture of the Proximal Metaphysis of the Tibia in Children," The Journal of Bone and
Joint Surgery, Vol. 62-B, 1980, p. 527.

[10] P. Bertland and S. Trillat, "Le Traitment de Inegalites des Longureur des Member's Inferieurs Pendant la Croissance," Ren Orthop, Vol. 34, 1984, pp. 264-311.

[11] K. P. Chanand and A. R. Hodgson, "Physiologic Leg Lengthening. A Preliminary Report," Clinical Orthopaedics, Vol. 68, 1970, pp. 55-62.

[12] B. G. Weber, "Fracture of the Proximal Tibial Metaphysis. In Treatment of Fractures in Children and Adolescents," Spinger, New York, 1980, pp. 324-329. 